\title{
Logic and Music in Plato's Phaedo
}

\author{
D. T. J. BAILEY
}

\begin{abstract}
This paper aims to achieve a better understanding of what Socrates means by

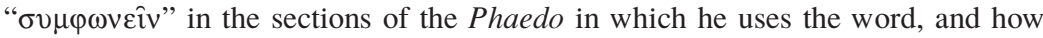
its use contributes both to the articulation of the hypothetical method and the proof of the soul's immortality. Section I sets out the well-known problems for the most obvious readings of the relation, while Sections II and III argue against two remedies for these problems, the first an interpretation of what the $\sigma v \mu$ -

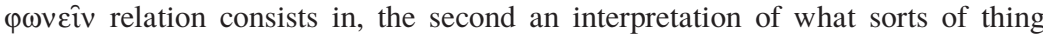
the relation is meant to relate. My positive account in Section IV argues that we should take the musical connotations of the term seriously, and that Plato was thinking of a robust analogy between the way pitches form unities when related by certain intervals, and the way theoretical claims form unities when related by explanatory co-dependence. Section V surveys the work of IV from the point of view of the initial difficulties and suggests further consequences for the hypothetical method, including the logical relation between the $\sigma v \mu \varphi \omega v \varepsilon i v$ and $\delta 1 \alpha \varphi \omega v \varepsilon i v$ relations, and the need for care in ordering the results of a hypothesis.
\end{abstract}

"But anyhow I proceeded in this way: on each occasion hypothesising the $\lambda$ ó which I judged to be strongest, I put down as true the things that seem to me to $\sigma \cup \mu \varphi \omega v \varepsilon i v$ with it - both about a causal account and any of the other things that are - but those things that did not I put down as false." (Phaedo 100a3-7).

"But if someone clung to the hypothesis itself, you would bid him goodbye and wouldn't answer him until you had examined its results, whether according to you they $\sigma 0 \mu \varphi \omega v \varepsilon i \hat{~ o r ~ \delta i \alpha \varphi \omega v \varepsilon i ̂ ~ w i t h ~ o n e ~ a n o t h e r . " ~(P h a e d o ~ 101 d 3-5) . ~}$

\section{The Difficulty}

These two passages from Socrates' Second Voyage in the Phaedo create a famous problem about how to understand what Socrates means by the

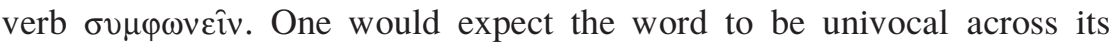
instances in the passages, partly because they appear so close together (an abrupt change in the use of the same word in such a short space of time seems unlikely), and partly because Socrates is here attempting to give a sense to what looks like the technical vocabulary of his new method. It is in giving a sense to technical vocabulary that one should be as selfconscious as possible about one's language: it would therefore be most unfortunate if we had to attribute ambiguous use to Socrates when he is 
doing just that. But, on the face of it, it seems that we must either impute an ambiguity to his use of $\sigma 0 \mu \varphi \omega v \varepsilon i v$, or give him a lunatic methodology that no one could possibly follow.

Let me start with the second passage. Socrates seems to be telling us not to go about giving a justification $\left(\lambda \text { ó }_{0}\right)^{1}$ for a hypothesis until we have examined its results (o $\rho \mu \eta \theta \dot{\varepsilon} v \tau \alpha)$ and seen that they are consistent with one another ( $\dot{\alpha} \lambda \lambda \dot{\eta} \lambda$ ors $\sigma \cup \mu \varphi \omega v \varepsilon \hat{\imath})$. Then and only then should we try to give an explanation of the hypothesis from which we got those results. That piece of advice makes good methodological sense, for the following reason. If you can show that inconsistent propositions follow from a hypothesis, that's enough for you to know straight away that the hypothesis is false - in fact self-contradictory - even though establishing that no inconsistency results is not sufficient to establish that the hypothesis is true. In other words, checking the results of your hypothesis for consistency is the best way to respond to the impatient interlocutor who wants a justification for the hypothesis as soon as you have made it. For the need to defend the hypothesis with a $\lambda$ ó ${ }^{\prime}$ os is surely abnegated if you find out that it is self-contradictory, which it will be if it yields inconsistent results. So checking your o $\rho \mu \eta \theta \dot{\varepsilon} v \tau \alpha$ for consistency offers the prospect of finding, as soon as possible, something sufficient for a final decision on the truth-value of your hypothesis: why cast about for a justification for a claim before you have satisfied yourself that the claim is not necessarily false?

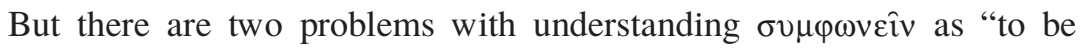
consistent with". Firstly, that is plainly not how Plato uses the word in other contexts, particularly Cratylus 436d1ff. Here Socrates and Cratylus agree that the etymologies discussed in the dialogue thus far indicate that names generally $\sigma 0 \mu \varphi \omega v \varepsilon \hat{i}$ one another by virtue of their all saying that their referents are in flux. Socrates goes on to argue that this kind of consistency is no guarantee of truth: for all the agreement among names discovered so far, the name giver might have been wrong from the start in supposing that the world is as Heraclitus said it is. But this relation among names, even though it cannot guarantee the correctness of any one of

\section{Accepted January 2005}

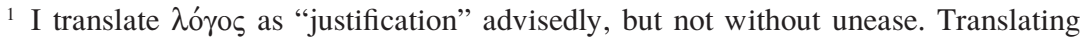
it as "proposition" here would surely be too weak: it cannot mean just any proposition, but a special kind of proposition, a justificatory one. But previously, in the famous

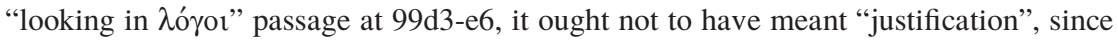
it is hardly illuminating to be told to look in justifications when one is searching for the truth about things. 
them, is surely stronger than the logical relation of consistency. A set of propositions is consistent if and only if there is no disagreement among its members: propositions having nothing to do with one another are consistent provided they could be true together. But when Socrates and Cratylus agree that all the names they have discussed so far $\sigma \cup \mu \varphi \omega v \varepsilon i$ one another, there is more to be said for them than that they lack disagreement. They have more in common than that by virtue of all telling the same story about the world.

But that the word crops up in other contexts meaning a relation stronger than mere consistency is a trifling difficulty compared with the second. The consequences are much graver if we suppose that Socrates means "to

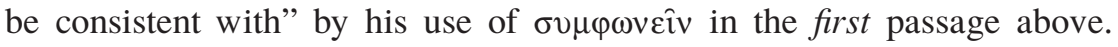
There Socrates tells us that he puts down as true whatever bears this relation to the hypothesis. But for any hypothesis, there will be an infinite number of propositions which are consistent with it, but which we have no independent reason to assert. Surely we are not to set these down as true simply because they bear a relation as weak as consistency to the hypothesis. It sounds ludicrous - and of course it results in disaster. For among this infinite number of propositions there will be pairs of propositions inconsistent with one another. So if Socrates means nothing

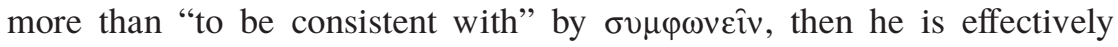
telling us in the first passage to assert contradictions, and his method is utterly crazy.

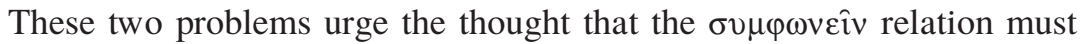
be stronger than that of consistency. So perhaps it is as strong as entailment. Socrates would then be telling us in the first passage to put down as true all and only those propositions that are entailed by the original hypothesis. In that case he would not be inviting us to assert propositions we have no reason to hold true: for indeed you do have good reason to accept $^{2}$ a proposition if it follows from a proposition you have already asserted. Nor will he be inviting us to assert contradictions in the first passage - or at least, if we are invited to assert a contradiction on the basis that one followed from the hypothesis, that would be sufficient for us to know that the hypothesis is necessarily false.

2 I need to write "accept" rather than "assert" here. After all, the billion-fold negation of "It's teatime" follows from "It's teatime". But just because I assert that it's teatime, I have no reason to assert its billion-fold negation, although I better had accept the latter as true whenever I do assert that it is teatime. 
But sadly this interpretation will not do either. For it fails to make sense

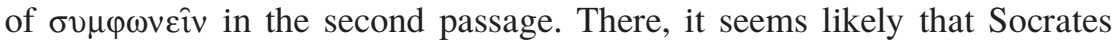
is talking of a relationship such that, if it holds between the results of a hypothesis, then that is reason to continue supposing the hypothesis true, but if it fails to hold, that is reason to suppose the hypothesis false. Whatever this relation is, it better had not be entailment, for it need not be the case that the results of true hypotheses entail one another. It is true that water is $\mathrm{H}_{2} \mathrm{O}$; from this, it follows that water is a compound; and it also follows that water contains oxygen. But neither of these results entails the other. A stuff need not contain oxygen in order to be a compound, while there is at least one stuff which contains oxygen without being a compound, namely oxygen itself, or at any rate a pure sample of it. If Socrates means "entail one another" by $\dot{\alpha} \lambda \lambda \dot{\eta} \lambda$ ors $\sigma v \mu \varphi \omega v \varepsilon \hat{\imath}$, then the results of the hypothesis that water is $\mathrm{H}_{2} \mathrm{O}$ do not $\alpha \lambda \lambda \eta \dot{\eta} \lambda$ ors $\sigma 0 \mu \varphi \omega v \varepsilon \hat{i}$. But that is no reason to deem false the hypothesis that water is $\mathrm{H}_{2} \mathrm{O}$.

And of course there is a more serious reason for rejecting the entailment reading, for it makes out that the first passage this time advocates the same outrageous promiscuity we found on the "consistency" interpretation. It was ludicrous to put down as true all the propositions consistent with the hypothesis because this involved us in, among other things, asserting contradictions. But it is also ludicrous to put down as false all the propositions which are not entailed by the hypothesis. For once again there will be an infinite number of them, including propositions we have independent reason to suppose true, and also contradictory pairs of propositions. Of course there is nothing wrong with putting down as false a conjunction of contradictory pairs: that is precisely what one ought to do. But it follows from what Socrates says in the first passage, on the entailment reading, that we will have to put down as false each member of a contradictory pair if neither is entailed by the hypothesis, and that is indeed disastrous. From the hypothesis that water is $\mathrm{H}_{2} \mathrm{O}$ it does not follow that I am in my office; nor does it follow, from the same hypothesis, that I am outside my office. But we had better not put down as false both these non-entailed propositions, for it cannot be that I am neither in my office nor outside it. ${ }^{3}$ So once again we have an interpretation that gives Socrates an unusable method.

3 It cannot be, of course, provided that we accept the Law of Excluded Middle, a more controversial principle than the Law of Non-Contradiction. But even so, supposing that Socrates might not subscribe to the Law of Excluded Middle is obviously much too high a price to pay in order to save the entailment reading, and would not 


\section{The Current View}

The best current solution to this problem is due to Jyl Gentzler. ${ }^{4}$ Reflecting on these problems for both the "consistency" and "entailment" interpretations of the $\sigma 0 \mu \varphi \omega v \varepsilon i v$ relation, she argues that Socrates must be talking about an interim relation, stronger than consistency but weaker than entailment: that is, a relation for which consistency is necessary but not sufficient, and for which entailment is sufficient but not necessary. A proposition q must be consistent with $\mathrm{p}$ in order to $\sigma \nu \mu \varphi \omega v \varepsilon \hat{\mathrm{i}} \mathrm{p}$, but that by itself is not enough for $\mathrm{q}$ to $\sigma \nu \mu \varphi \omega v \varepsilon \hat{\mathrm{i}} \mathrm{p}$; if $\mathrm{q}$ is entailed by $\mathrm{p}$, then it will $\sigma \nu \mu \varphi \omega v \varepsilon \hat{\mathrm{i}} \mathrm{p}$, although it need not do the former in order to do the latter.

In what follows I shall be arguing that Gentzler is basically right in thinking that the relation lies somewhere between consistency and entailment, but the devil is in the details that need spelling out in showing what that involves, and in relating those details to the hypothetical method in general and the argumentative context in particular. Gentzler's details, sadly, do not add up to a genuine relation: for some propositions can perfectly well entail others with which they are inconsistent, namely their own negations. In fact Gentzler's relation will both hold and not hold between a contradiction and its negation, as I shall show. To put the point in context, if Socrates means Gentzler's relation when he uses the word $\sigma v \mu-$

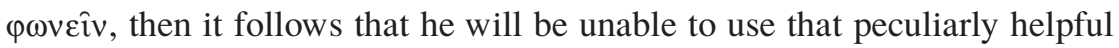
style of reasoning, so beloved of Greek mathematicians, known as arguing by reductio ad absurdum, which exploits the properties of such propositions. When we argue by reductio ad absurdum, we give grounds for supposing a proposition false because it entails a contradiction, in which case it is self-contradictory. Now Gentzler's relation is such that q has to be consistent with $\mathrm{p}$ if $\mathrm{p}$ entails it, given that entailment is sufficient but consistency necessary for $\mathrm{p}$ to $\sigma \nu \mu \varphi \omega v \varepsilon \hat{i}$ with $\mathrm{q}$. But what about the situation in which $q$ is none other than $\neg$ p? If Gentzler's Socrates were to find out that his hypothesis entailed its own negation - that is, if he were to refute it by reductio ad absurdum - then far from doing the sensible thing and rejecting his hypothesis as false because self-contradictory, he would have to conclude that he had made an invalid inference somewhere along the way. For the fact that $\mathrm{p}$ and $\neg \mathrm{p}$ are inconsistent with one another would

by itself be sufficient to save that reading anyhow, for Socrates would still be telling us to deny many things we have no reason to deny.

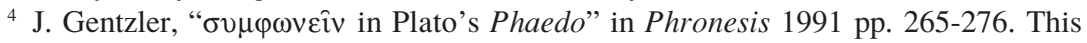
paper owes a good deal to Gentzler's presentation of the issues. 
be enough to indicate to anyone supposing there was such a relation as Gentzler's that $\neg$ p doesn't really $\sigma v \mu \varphi \omega v \varepsilon \hat{i}$ (in the sense of "follow from") p! By such a method, Socrates could never find out if his hypothesis was self-contradictory: his method would therefore be utterly useless. ${ }^{5}$

An example might help here. Take the hypothesis that heavier bodies fall faster than lighter ones. That claim sounded safe and strong to no less a thinker than Aristotle. Now imagine a cannon ball and a ping-pong ball falling. It follows from the hypothesis that if we tie the two together, the product will fall more slowly than the cannon ball alone, since the pingpong ball, by itself falling more slowly, will act as a drag on the cannon ball. But since the conjunction of the cannon ball and the ping-pong ball will be heavier than the cannon ball alone, even if only by a little, it also follows that it will fall faster than the cannon ball alone. So a cannon ball tied to a ping-pong ball will fall both faster and slower than a cannon ball alone: we have a contradiction. Now the lesson Galileo famously drew at this point was that the initial Aristotelian hypothesis is false, simply because it entails this contradiction, and hence all bodies must fall at the

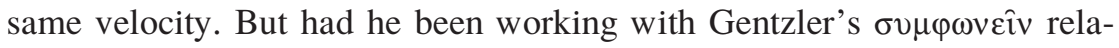
tion, he could not have drawn this eminently sensible conclusion. For on realising that the conclusion is inconsistent with the hypothesis - for contradictions are inconsistent with every proposition - he would have inferred that it does not $\sigma 0 \mu \varphi \omega v \varepsilon i$ the hypothesis. In that case, it is not entailed by it, for entailment is sufficient for the $\sigma \nu \mu \varphi \omega v \varepsilon i v$ relation. Therefore

5 In correspondence, Andrew Barker has suggested a riposte to this argument: Socrates might still work with a relation such as Gentzler's because in calling his hypothesis "the strongest" in the first place, presumably he indicates that he is confident that it is not self-contradictory. I reply simply that such confidence would of course be no guarantee that in fact his hypothesis is not self-contradictory, any more than Frege's confidence that Axiom V was "purely logical" (Preface to Grungesetze der Arithmetik Vol. 1 (1893)) was enough to save it from Russell's paradox. The comparison with Frege aside, Barker's defence of Gentzler seems to be prima facie implausible on the following grounds: the whole point of calling something a hypothesis in the first place is to indicate its provisionality, the fact that it is not yet known for all that it is supposed, and is to that extent revisable. But if a proposition is treated as revisable in principle, pending the results of its theoretical development, why should we suppose that it is nonetheless exempt from the particularly violent form of revision involved in rejecting a previously accepted proposition on the grounds that it is self-contradictory? If a proposition is hypothesised on the grounds that it might turn out to be false, surely one should allow that it might turn out to be false in any way propositions can be false, including being false by virtue of being self-contradictory. 
he would have racked his brains for where he went wrong in pursuing the o $\rho \mu \eta \theta \dot{\varepsilon} v \tau \alpha$ of Aristotle's hypothesis, and mechanics would be all the poorer. Certainly he would not have been justified in inferring that the Aristotelian hypothesis is false.

Of course it is one thing to complain that there is something methodologically wrong with the relation Gentzler reads into Socrates' use of

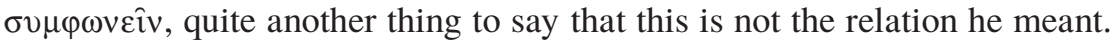
The considerations above about consistency and entailment are somewhat refined, and may well not have occurred to Plato, for all that his probable familiarity with mathematics would have put him in the way of countless reductio proofs (not to mention the variety of such arguments in the dialogues). But for the moment I think that charity requires that we look for an alternative account of the relation if one is available: and, even prescinding from this problem, there is another motive for seeking a new interpretation in any event. In order to work out what is going on here to our satisfaction, our focus must not be restricted to the $\sigma \nu \mu \varphi \omega v \varepsilon i v$ relation, whatever it is, alone. Socrates is here giving us a criterion for deciding on the truth and falsehood of propositions, and it is much too much to hope that the relation he invokes will allow us to make truth-evaluations of any proposition whatsoever relative to the hypothesis. So in order to get to his meaning, then at the same time as we are considering the

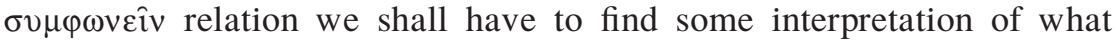
things - what class of propositions - we are supposed to classify as true or false according to whether they bear the $\sigma 0 \mu \varphi \omega v \varepsilon i v / \delta 1 \alpha \varphi \omega v \varepsilon i v$ relations to our original hypothesis. It is just this that is missing both from the obviously problematic interpretations of the $\sigma \nu \mu \varphi \omega v \varepsilon i v v$ relation, but also from Gentzler's interim solution.

\section{Consistency on a Subject Matter}

One possibility for answering the second problem above is that the right candidates for satisfying those relations will be propositions on the same

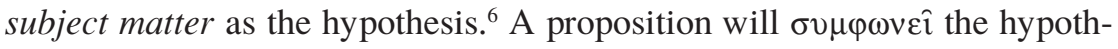
esis if it is consistent with it and on the same subject matter; it will

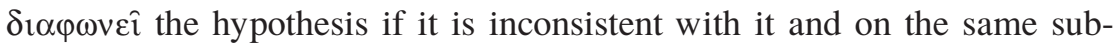
ject matter; and if it is not on the same subject matter, it is not even a candidate for satisfying either the $\sigma v \mu \varphi \omega v \varepsilon i v$ or $\delta 1 \alpha \varphi \omega v \varepsilon i v$ relations with 
the hypothesis. Such a reading might have a rather attractive feature: provided you take into consideration the requirement about being on the same subject matter, the $\sigma u \mu \varphi \omega v \varepsilon i v v$ and $\delta 1 \alpha \varphi \omega v \varepsilon i v v$ relations amount unproblematically to consistency and inconsistency. In effect, the constraint that the candidate propositions be on the same subject matter as the hypothesis synthesises the rival interpretations above which made Socrates' theory out to be ridiculous: for any proposition consistent with a hypothesis and on the same subject matter should also be entailed by that hypothesis, provided we construe "subject matter" in a sufficiently fine-grained fashion. ${ }^{7}$

But this will not quite do as it stands, for the opacity of the relations between different propositions on the same subject will make proceeding with such a method practically impossible. For example, Socrates explicitly says that the subject matter of his hypothesis is "a causal account"

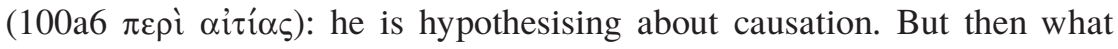
attitude is he supposed to take, for instance, to another famous ancient claim about causation, Aristotle's claim that there are four causes? Is that claim consistent or inconsistent with Socrates' hypothesis? It certainly seems to be a candidate for such a question, given that it is apparently about the same subject matter, causation. But here we are in danger of reasoning as follows: "Aristotle's claim is on the same subject matter as Socrates'; there is no detectable inconsistency between the two, at this stage ${ }^{8}$ so on these grounds we should suppose Aristotle's claim true." Obviously, this would be a disastrous method of proceeding which would involve us in asserting many falsehoods, simply because these means for deciding on the truth-values of propositions are just too slender. But if instead we reason that Aristotle's claim is not yet a candidate for bearing the $\sigma v \mu-$

7 There would still be a problem, I think, with the results of a hypothesis entailing one another. "Water is a compound" and "Water contains oxygen" are both propositions consistent with one another and on the subject "chemical composition of water": but they do not appear to entail one another, as I argued above. It might be that this problem could be overcome by some suitable tweaking of the notion of a subject matter: one might argue that the two propositions are not really on the same subject matter, but that the former is about the chemical structure of water while the latter is about the quite different subject of its constituents. But even if such a move could be made successfully, it would be highly artificial and ad hoc; and I think the further objections above are quite decisive against the subject matter interpretation.

8 A sign of this, of course, is the vast scholarly literature debating whether the theory of causation at the end of the Phaedo countenances final, efficient and material causes as well as formal ones. This debate could not have occurred if we could tell on the face of it that Aristotle's claim is inconsistent with Socrates' hypothesis. 
$\varphi \omega v \varepsilon i v / \delta 1 \alpha \varphi \omega v \varepsilon i v$ relations to the hypothesis, then on the present interpretation there is no explanation for this - why is it not yet a candidate, given that it is at least about the same subject?

There is an even more serious problem with speaking of subject matters in this context. Intuitively, it seems that a subject matter is determined by the fact that such-and-such propositions are all the right ones. If you were to ask me "What is the subject matter of Chemistry? What is it all about?" then I suppose I should best answer you by showing you an elementary Chemistry textbook - that is, by showing you a well-organised collection of true propositions. On the other hand, this thought cannot be quite correct: for when a boy makes a mistake in his Chemistry exam by failing to balance his equation properly, it is not as if he has gone off the subject altogether and started writing about a different topic such as history or botany. Our intuitions conflict here. Take the once-believed proposition "There is a planet Vulcan between Mercury and the Sun". Is that a proposition about astronomy? Is it on the same subject matter as the truth "There are nine planets in the solar system"? Well ... yes and no. In a way it is about astronomy because it tries to talk about the things astronomy deals with, planets and so forth. But in a way it is not about astronomy because it is false: there is no such planet as Vulcan, so what it purports to speak of is not one of the objects of astronomy.

I infer from all this, even with the qualification that our intuitions somehow conflict, that the notion of a subject matter is just a little too close to the concept of truth for it to be entirely helpful in explicating Socrates'

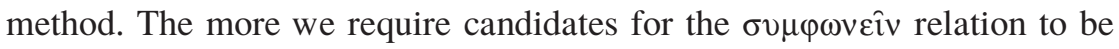
on the same subject matter as a hypothesis, the more we require them to be true - that is, the more we deny ourselves the possibility of explaining how they can be false. And we should not want to deny ourselves that possibility. For one thing, as I have just said, Socrates' method has got to be one with which we can proceed. It is a constraint on any usable heuristic procedure, which Socrates' method is surely meant to be, that we must be able to use it in advance of knowing the right answers, of knowing what is true and what is false. Socrates must not be made out to be saying "Start from a true hypothesis, and then set down as true any propositions that are materially equivalent with it": for that advice is no more helpful to a philosopher than the recommendation "Bet on black when the ball is going to land on black, and bet on red when it will land on red" is to a roulette player. ${ }^{9}$ We must be able to move forward even 
from a false hypothesis, which means we must be able to suppose that falsehoods $\sigma v \mu \varphi \omega v \varepsilon i$ the hypothesis without thereby failing to follow the method. How can we do that on the present account if falsehoods do not, in some important sense, have a subject matter?

\section{The Musical Connection}

My own tack for responding to the problem of the $\sigma 0 \mu \varphi \omega v \varepsilon i v$ relation involves examining the logically promising features of the musical systems suggested by the word, and seeing if appropriate analogues for them can be found in good scientific theories. That we should look for inspiration in music to help us here, and suppose that Plato might have done so as well, is not at all perverse. The Phaedo is a dialogue thoroughly soaked in musical allusions above and beyond the connotations of the word $\sigma v \mu-$

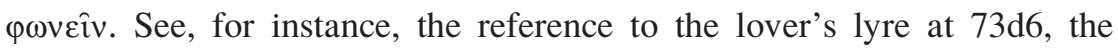
request for a "soothing song" at $77 \mathrm{e} 8$ ( $\dot{\varepsilon} \pi \alpha \underline{\alpha} \delta \varepsilon \imath v$, echoed at $114 \mathrm{~d} 7$ ), Simmias' hypothesis that the soul is an attunement ( $\alpha \rho \mu o v i \alpha)$ at $85 \mathrm{e} 5 \mathrm{ff} .{ }^{10}$ and so on. It should come as no surprise to find Socrates drawing detailed philosophical inspiration from music and musical theory in a dialogue so dominated by the figure of Apollo. ${ }^{11}$

Musical systems should interest us in this context because, at least at first glance, they exhibit some of the properties we find in good theories. That is, they are complex, well integrated systems whose elements (propositions in the one case, pitches in the other) agree with one another in

hypothesis must satisfy: for one might just as well say "Proceed by putting down as true all and only propositions that are materially equivalent with ' $2+2=4$ '”.

${ }^{10}$ The theory that the soul is an attunement may have originated from the Pythagorean philosopher and harmonic theorist Philolaus, to whom there is an allusion at Phaedo 61d6-7. For further details see A. D. Barker, Greek Musical Writings Volume II: Harmonic and Acoustic Theory (Cambridge 1989) pp. 38-9. Everything I have to say about Greek music owes a great deal to this work, as well as its author's generous and invaluable correspondence.

${ }^{11}$ If more justification is needed, I refer the reader to M. F. Burnyeat, "Plato on Why Mathematics is Good for the Soul" in T. Smiley ed. Mathematics and Necessity (London 2000) pp. 1-81. Burnyeat argues very plausibly that the key to understanding the connection between the content of mathematics and the moral value that a mathematical training is supposed to impart to the rulers of the ideal city, according to the central books of the Republic, is to be found in the study of harmony (e.g. p. $47 \mathrm{ff}$.). In other words, the real philosophical nature of the hypothetical method par excellence, mathematics, is to be explicated best by an investigation of musical theory. That is exactly what I am suggesting for the hypothetical method in the Phaedo. 
some important sense, ${ }^{12}$ while also standing in significant and determinate relations to one another. A musical $\dot{\alpha} \rho \mu$ oví $\alpha$ is not just a bunch of different pitches, just as a correct theory is not just a mass of true propositions - in both cases the ordering of, and relations between, the elements is important. ${ }^{13}$

The idea that musical systems are significantly structured is not something I have lifted anachronistically from contemporary diatonic music. It lay at the heart of Greek music as well. Its origins appear to lie in the thought that pitches related by certain intervals ${ }^{14}$ - the octave, the perfect fifth and the perfect fourth - blend together to form a unified whole, a $\sigma v \mu \varphi \omega v i \alpha$, while notes that are otherwise related do not form such integrations. ${ }^{15}$ The various familiar species of Greek $\alpha \rho \mu o v i ́ \alpha 1$, Dorian, Mixolydian and so forth, are then constructed by permuting other pitches in various ways on to a basic structure of $\sigma v \mu \varphi \omega v i \alpha 1$. $\alpha \rho \mu$ ovía are therefore ordered structures of pitches constituted by relations of different strengths: weak relations which must hold between all the notes in a

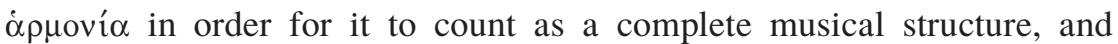
stronger relations which hold between certain pitches in the $\alpha \rho \mu$ oví $\alpha$ which are sufficient for those pitches to form $\sigma v \mu \varphi \omega v i \alpha$. It is the latter that especially concern us in explicating my view of the Phaedo's concept of $\sigma 0 \mu \varphi \omega v \varepsilon i ̂ v$.

Plato gives us a characterization of these special intervals in the Timaeus, when he has Timaeus argue that different pitches succeed in

12 See Symposium 187b4-5, where Erixymachus says that musical harmony is a kind

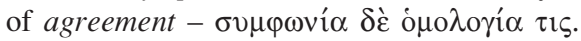

${ }^{13}$ It is also worth bearing in mind the fact that musical systems and collections of

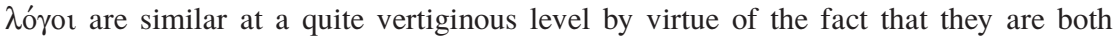
somehow imitative. The imitative nature of $\lambda$ ó yor is enough, in the Phaedo, to recommend them as the objects of investigation for the hypothetical method we are discussing. The imitative nature of the $\alpha \rho \mu$ oví $\alpha$ is enough to recommend the thought, adumbrated in Republic 397a-401b, that they must be restricted and regimented in the ideal city lest in aping undesirable emotions they do a disservice to the education of the young.

14 More precisely: pitches related by intervals expressible in terms of ratios that are either multiple $(n: 1)$ or epimoric $(n+1: n)$. This seems to be the closest one can get to a general characterisation of the $\sigma v \mu \varphi \omega v i \alpha$, although it expresses neither necessary nor sufficient conditions for being a $\sigma v \mu \varphi \omega v i ́ \alpha$. Not all such ratios express $\sigma v \mu \varphi \omega v i ́ \alpha$ (e.g. 3:1); and at least one interval forming a $\sigma v \mu \varphi \omega v i \alpha$, the octave plus fourth, cannot be expressed either as multiple or epimoric (its ratio is 8:3).

15 One of these intervals is actually mentioned by name when Socrates is illustrating the "subtle" things that will not receive opposites although they are not themselves opposites. Among this class, we read at $105 \mathrm{~b} 1$, is $\tau$ ò $\dot{\eta} \mu$ tó $\lambda$ tov, the ratio of the fifth (3:2). This is yet further evidence that Plato has music in mind in our passage. 
forming $\sigma v \mu \varphi \omega v i \alpha$ when "by attaching a similarity, they blend together a single experience out of high-pitched and low-pitched movement"

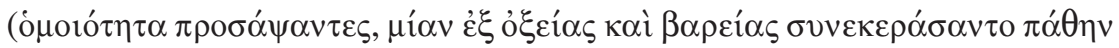
80b4-5). Aristotle was also interested in the $\sigma 0 \mu \varphi \omega v i \alpha 1$, giving them a very similar description at De Sensu 448a9-11. Pitches standing in appropriate ratios to one another form a mixture whereby each note is perceived

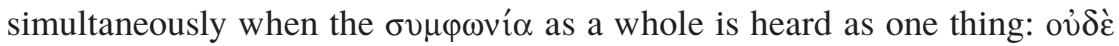

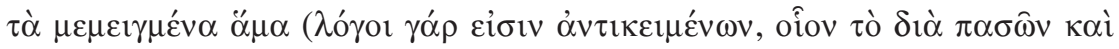

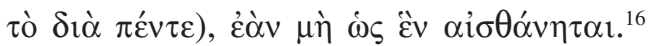

Now the precise musical details need not concern us here. ${ }^{17}$ All we need is the thought that, within a musical structure, a $\alpha \rho \mu$ oví $\alpha$, certain pitches are so related to one another that they form some kind of unity, which notes that are not so related do not form. When notes are in the ratios

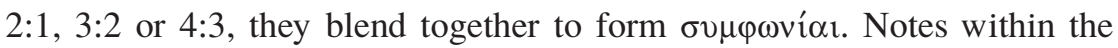
$\dot{\alpha} \rho$ oví $\alpha$ that are not so related do not form this strong unity together, although they are still part of the same structure. Is there a relation hold-

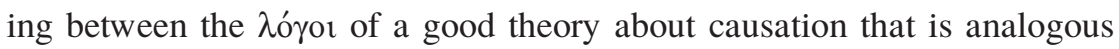
to this special unifying relation holding between the pitches in a $\sigma v \mu-$

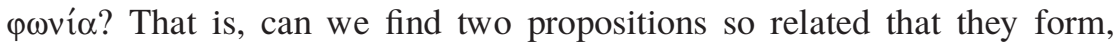
as it were, some kind of unity over and above their being merely asserted together, just as two pitches in the ratio $2: 1$ or $3: 2$ blend to form a $\sigma v \mu-$ povía (an octave or a fifth) over and above their being each sounded?

I think there is enough information in the Phaedo to suggest that Socrates' hypothesis and its results might stand in such a relation to one another - a relation sometimes discussed in recent philosophy of science. ${ }^{18}$ This relation holds between propositions if they stand as explanations of

16 Both these passages are discussed in A. D. Barker, Op. Cit. pp. 76 \& 62 respectively.

17 This is convenient: for if they did then we would need an argument about the extent of Plato's detailed knowledge of harmonic theory, which would be extremely difficult to formulate. M. F. Burnyeat, Op. Cit. p. 15ff., argues that he was at least familiar enough with the writings of the Pythagorean harmonic theorist Archytas of Tarentum to quote him (consciously or unconsciously) at Republic 530d8.

18 I have in mind: P. Kitcher, "Two Approaches to Explanation" in Journal of Philosophy LXXXII 1985 pp. 632-639; W. Salmon, "Scientific Explanation: Causation and Unification" in Causality and Explanation (Oxford 1998) pp. 68-78. The example I use is taken from Salmon. A more general statement of the view that explanations can both explain something and be themselves explained by it is to be found in W. V. O. Quine and J. Ullian, The Web of Belief (New York 1970) p. 79: “. . there can be mutual reinforcement between an explanation and what it explains. Not only does a supposed truth gain credibility if we can think of something that would explain 
one another, albeit in different ways. Take the two propositions "Copper is a conductor" and "Pennies conduct electricity". These claims form a kind of unity because they provide one another with mutual explanatory support. The claim that copper is a conductor stands as an explanation for the claim that pennies conduct by being more general than the latter: it says what it is about pennies that makes them conduct, and that what pennies are made of conducts whether it is in penny form or not. One might similarly explain in turn the claim that copper is a conductor with the claim that metals are conductors. Again, we say what it is about copper that makes it a conductor. And one might again explain this proposition with the claim that electrons in such-and-such a relation conduct electricity, and so on. I shall call this generalising explanation: ${ }^{19}$ we explain particular facts by subsuming them under more and more general laws "higher" principles, as Plato would call them. But there is also what I shall call particularising explanation, which seeks to explain away the most general explanatory formulae by characterizing such truths as laws about causal relations between particular things. The idea is that the laws are shown to be true by virtue of the explanatory support they get from their instantiations. In this way, we can give a kind of explanation for the claim that copper is a conductor by pointing out that there are particular copper things, namely pennies, and that they all conduct electricity. ${ }^{20}$

I think that both these kinds of explanation are on display in the Phaedo. Socrates' starting point is the general one about the causal power of Forms, which we might represent schematically as "There is such a

it, but also conversely: an explanation gains credibility if it accounts for something we suppose to be true."

${ }^{19}$ Kitcher and Salmon call this style of explanation "bottom-up", referring to the direction we move in going from the explanandum to the explanans. But many philosophers use "top-down" as an expression for explanation of the particular by the more general. Hence I avoid the terminology altogether.

${ }^{20}$ I have occasionally encountered resistance to the thought that "Pennies conduct" is any kind of explanation of "Copper is a conductor". Perhaps the example is too trivial to express the point. Certainly I grant that it is not a generalising explanation of the latter; and I grant too that in science we are often more interested in generalising explanations than other kinds since it is the particulars with which we are already familiar through perception. But these concessions are neither individually nor jointly sufficient for scepticism about the possibility of particularising explanations. It is mere question begging to suppose either that generalising explanations are the only explanations, or that whenever we want an explanation we already have a grasp on some quite particular fact and are casting about for a more general one. 
thing as the $\varphi$, and if anything else happens to be $\varphi$, then it is $\varphi$ by the

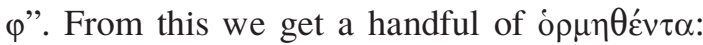

1. When something is $\varphi$, then $\varphi$-ness is somehow in that thing.

2. Anything that is $\varphi$ by the $\varphi$ is different from that by which it is $\varphi$. (It is by Simmias' largeness that he overtops Socrates, not by his nature.)

3. If $\varphi$ and $\psi$ are opposites, then the $\varphi$ or $\psi$ in things will not tolerate the advance of the other: either it will retreat or perish on the approach of its opposite.

It seems to me that in moving from the original hypothesis to 1, 2 and 3, we get successive particularising explanations: 1, 2 and 3 explain how Socrates' hypothesis can be true by exploring what happens in particular applications of that very general claim. 1 for instance explicates the details of what is meant by " $\varphi$ by the $\varphi$ ": when something is " $\varphi$ by the $\varphi$ ", that is because something of the $\varphi$ is in that thing. Likewise, 2 gives some particularising explanation for 1: it says a little about how the agents of causation are in their subjects. Since they are different from their subjects, they are in them without being them. And in turn 3 provides further explanation for 2 by suggesting that the domain over which the variable $\varphi$ ranges, containing the properties that are in things without being them, is populated by things that have opposites. The things that can have $\varphi$ in them - Simmias' size, Socrates' fingers, Phaedo's face - are not the sort of things that have opposites: this is why such things must be different from the causal agents in them, as 2 asserts. 2 gives particularising explanation for 1 while 3 explains 2 in a similar fashion.

But equally, we have generalising explanations here. Suppose someone were to ask "How is it that certain properties of a thing are capable of doing such things as advancing, retreating or perishing? That is, how is it that 3 is true?" We can explain this with the more general 2: certain properties are capable of doing such things because they are different from the things that have them, and hence can advance into or retreat from the hosts from which they are different without impairing their identity. We can explain this in turn by ascending to 1 . Things have properties that are different from themselves while still characterising them when they have them because, when they have them, those properties are somehow in them. And if we ask for a more general explanation of this fact, we get it from Socrates' original hypothesis: properties are in things because, more generally, when something other than the $\varphi$ itself is $\varphi$, it is so in 
virtue of, because of, something else, the $\varphi$. In fact Socrates is quite explicit that he intends to reach his conclusion using this kind of generalising explanation, when he says at 100b7-9 that $\ddot{\alpha} \varepsilon \iota^{\prime \prime} \mu o r \delta i \delta \omega \varsigma \tau \varepsilon \kappa \alpha i$

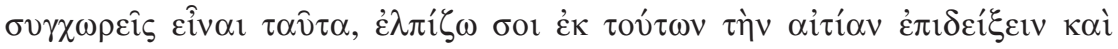

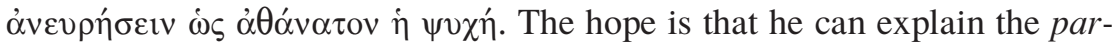
ticular fact that the soul is immortal by showing how it is related to truths about coming to be and passing away generally.

I have been arguing that the same sort of relationship holds between Socrates' hypothesis and its various o $\rho \mu \eta \theta \dot{\varepsilon} v \tau \alpha$, taken as pairs of propositions, as holds between the propositions "Copper is a conductor" and "Pennies conduct electricity". As pairs of propositions, they form a unity in virtue of supporting one another in different ways, by providing different kinds of explanation, generalising or particularising, for one another. Both kinds of explanation hold, in different directions, between the hypothesis and 1, 1 and 2, and 2 and 3. And this is strongly analogous to the way in which pairs of pitches form a unity by blending with one another. Two notes an octave apart do not bear the same relation to one another. For while blending is a symmetrical relation, the precise relation of lying in such and such a ratio to another pitch is asymmetrical. ${ }^{21}$ Likewise, the propositions about copper and pennies above do not bear the same relation to one another. For while explanation, as I have argued, is a symmetrical relation, the precise relations of generalisingly explaining or particularisingly explaining are asymmetrical. And in both cases, the musical and the theoretical, something new emerges from the combination of the two things so related - a blended unity - which does not emerge, for instance, when we sound a pitch together with another six semi-tones above it, or when we conjoin truths such as "Cats are mammals" and

21 This is not an end to their logical similarity. In addition to being asymmetrical, concordant relations are irreflexive - no pitch blends into a $\sigma u \mu \varphi \omega v i ́ \alpha$ with itself and non-transitive, in that in some cases where pitch a blends with b and b with c, a will also blend with $\mathrm{c}$ (as when $\mathrm{b}$ is a perfect fifth above a and $\mathrm{c}$ a perfect fourth above $b$, where $c$ will be an octave above a), but in other cases a will blend with $b$ and $\mathrm{b}$ with $\mathrm{c}$ without a also blending with $\mathrm{c}$ (I give an example of this later on in the main text). These logical properties also hold of explanation. The kind of explanations in which I am interested are irreflexive: the proposition $\mathrm{p}$ is surely neither a generalising nor a particularising explanation of itself (although I grant that some propositions can, in some sense, be self-explanatory). Such explanations are also nontransitive, in that sometimes where $\mathrm{p}$ explains $\mathrm{q}$ and $\mathrm{q}$ explains $\mathrm{r}$, $\mathrm{p}$ will explain $\mathrm{r}$, but sometimes not. 


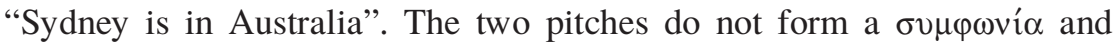
blend into a musical unity, just as the two propositions do not blend into an explanatory unity.

\section{The Solution proves its worth}

At this point one might object that the musical analogy amounts to a mere interesting observation rather than a plausible theory about how Plato might have understood the special kind of agreement that occurs between propositions when they $\sigma u \mu \varphi \omega v \varepsilon \hat{\imath}$ one another. It might be that Plato has Socrates develop his hypothesis in the way in which he does without ever thinking of musical $\sigma 0 \mu \varphi \omega v i \alpha$ at all, and any workable analogy with concordant relations in musical theory is pure coincidence. And I admit that this is perfectly possible. But let me restate the case for the analogy now that I have explicated its non-musical aspect. It seems to me that we need an answer to the following question. Given the likelihood that Plato was asking himself "What more is required of a proposition besides consistency with the hypothesis if it is to count among that hypothesis' o $\rho \mu \eta \theta \dot{\varepsilon} v \tau \alpha$ ?", to what kind of structure would he turn his mind for an answer? It seems likely that he would first think of a mathematical structure, given that mathematics is the most obvious example of a science that reasons from hypotheses, as Socrates takes himself to be doing in our passage. But if he was thinking of mathematics, he would almost certainly have thought of harmonic theory, especially since music has already been such an important theme in the Phaedo. And if he was thinking of harmonic theory, he might well have been struck by the way certain privileged intervals form stronger and more interesting relations between their pitches than other equally musical intervals within the $\alpha \rho \mu o v i \alpha$, just as some propositions form stronger and more interesting relations with propositions they explain, and are explained by, than other propositions equally consistent with them.

Let us turn once again to the problems facing the method outlined above to see if the new interpretation overcomes them. We saw that there was a problem about cashing out $\sigma 0 \mu \varphi \omega v \varepsilon i ̂ v$ solely in terms of consistency and "being on the same subject matter" as a hypothesis. We wondered how one could arbitrate between propositions on the same subject matter as a falsehood, because it isn't clear how a false proposition can have a subject matter. Things are much clearer when we deal instead with the notion of explanation. For it is perfectly possible for a proposition to be consistent with another and explanatory of it, and vice versa, even 
when both are false. My explanation takes into account the possibility of someone asserting a false hypothesis and then mistakenly putting down as true falsehoods that genuinely $\sigma \nu \mu \varphi \omega v \varepsilon \hat{i}$ it without thereby failing to follow the method. In other words I can clearly explain how we can proceed with Socrates' method without already having the right answers, which the "subject matter" interpretation could not - which means I can continue to hold for the moment that the hypothetical method is genuinely heuristic.

Again, it was not clear what we should do with a proposition on the same subject matter as a hypothesis, but neither clearly consistent nor inconsistent with it. Aristotle's claim that there are four causes bore this relation to Socrates' hypothesis. But if Socrates is looking for propositions that are consistent with his hypothesis but also explain it and are explained by it, then it seems we are on safer ground. For while we may not be able to tell initially whether a proposition on the same subject matter as our hypothesis is consistent or inconsistent with it, we can tell with much greater ease whether the proposition bears an explanatory relation to the hypothesis or one of its results. Explanation is much more transparent to our minds than consistency, as the career of Frege testifies. Since Aristotle's claim that there are four causes does not seem to cohere in the right way with either the hypothesis or the results mentioned so far, that is a sufficient reason not to put the claim down as true. But since there is not any detectable inconsistency either, we ought not to put it down as false. So it must be one of the infinite number of propositions which is not a candidate for either of the $\sigma 0 \mu \varphi \omega v \varepsilon i ̂ v / \delta 1 \alpha \varphi \omega v \varepsilon i ̂ v$ relations. Here again this interpretation scores well against the "subject matter" one described above. There is no danger of supposing Aristotle's thesis true simply because it is on the same subject matter as the hypothesis and not detectably inconsistent with it: for it does not adequately explain it. We also have a general answer to the question "what more is required from a proposition about a certain subject matter for us to be able to proceed with it (i.e. make a decision about its truth-value) rather than temporarily lay it aside and cast about for others?" The account says this: we can proceed if and only if it is either detectably inconsistent with the hypothesis, or both consistent with it and a good explanation of it in the sense elucidated. If a proposition satisfies neither of these conditions, that is sufficient reason for supposing that it cannot yet satisfy the $\sigma v \mu \varphi \omega v \varepsilon i v / \delta 1 \alpha \varphi \omega v \varepsilon i v$ relations, and hence is not yet a candidate for truth-evaluation.

From this we can also derive the interesting result that the $\delta 1 \alpha \varphi \omega v \varepsilon i v$ relation is the contrary, not the contradictory, of the $\sigma v \mu \varphi \omega v \varepsilon i v v$ relation. 


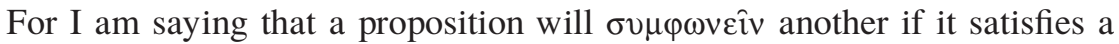
conjunction of conditions: it must be consistent with it and explain it. Since the negation of a conjunction is the disjunction of the negated conjuncts, then any proposition will satisfy that relation relative to another if it is either inconsistent with it or fails to explain it in the right way. So if the $\delta \alpha \varphi \omega v \varepsilon i v$ relation were the contradictory of $\sigma v \mu \varphi \omega v \varepsilon i v$, then we would once again be lost in a ruinous wilderness, putting down as false all those propositions which fail to explain our hypothesis, which will once again include propositions we have independent reasons to suppose true and, worse, contradictory pairs. Therefore the $\delta \alpha \varphi \omega v \varepsilon i v$ relation had better be its contrary, consisting simply in inconsistency with the hypothesis or one of its results. Put another way, the $\delta \alpha \varphi \omega v \varepsilon i v$ relation is not the negation of the $\sigma 0 \mu \varphi \omega v \varepsilon i v$ relation, but its opposite. This should come as no surprise at all given that Socrates' Second Voyage embodies the most opposition-fixated passage of an opposition-fixated dialogue.

The musical analogy also helps us to give a little colour to the "yet"s at the end of the last paragraph but one. As I have said, we have to suppose at least initially that Aristotle's claim that there are four causes is not yet a candidate for the $\sigma 0 \mu \varphi \omega v \varepsilon i v / \delta 1 \alpha \varphi \omega v \varepsilon i ̂ v$ relations. But if we are to have a fully developed theory of causation it surely must become one at some point. After all, a completely satisfactory theory of causation must be one that says, among other things, how many sorts of causation there are. In fact we have to suppose generally that in the development of a theory a large number of propositions start out beyond the pale of consideration but become candidates later on. Fortunately, exactly the same thing can happen in music. To illustrate this, let us simplify things by putting the point in modern terms. Take, for instance, a note of pitch $\mathrm{C}$, which is going to be the starting pitch and basis for a scale or attunement just as an initial hypothesis is the starting point and basis for a theory about something. The $\mathrm{C}$ an octave higher will form a $\sigma v \mu \varphi \omega v i \alpha$ with it. So will the $\mathrm{F}$ a fourth above middle $\mathrm{C}$ and the $\mathrm{G}$ a fifth above it. What about the $\mathrm{D}$ that lies a tone above the starting point? It does not form a

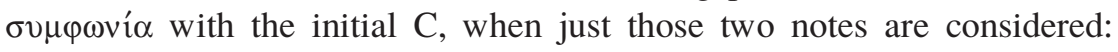
tones are not $\sigma v \mu \varphi \omega v i \alpha 1$. But it does form a $\sigma v \mu \varphi \omega v i \alpha$ with the $\mathrm{G}$ a fifth above $\mathrm{C}$, since it lies a perfect fourth below it. Hence, as the complex of relations is developed further and further, new notes form $\sigma v \mu \varphi \omega v i \alpha$ within the system that were incapable of forming such unities earlier when the right pitches with which to blend were not yet available. $\mathrm{C}$ does not blend with D by itself. But since $\mathrm{C}$ blends with $\mathrm{G}$, and $\mathrm{G}$ blends with $\mathrm{D}$, 
the structure CGD forms a unity held together by the middle term, G. ${ }^{22}$ And one can imagine the same sort of thing happening to Aristotle's claim. It does not blend with Socrates' hypothesis or any of the results adumbrated in our section of the Phaedo because it does not explain any of them in the right way. But it may well be that it will blend with propositions added later on in the development of the theory, which themselves blend explanatorily with earlier results, in which case it ought to be counted as true.

This musical understanding of $\sigma u \mu \varphi \omega v \varepsilon i v v$ also helps us, I think, to understand better why Socrates is so keen to stress the value of order when he concludes the introduction of the method that relies upon this relation. Consideration of the explanatory relations I have been using to characterize the meaning of $\sigma v \mu \varphi \omega v \varepsilon i v$ sharpens the issue. For it seems to be perfectly possible that two propositions could be consistent with your hypothesis and explanatory of it, without bearing that relation to one another. ${ }^{23}$ For instance, starting from the hypothesis "Metals conduct electricity", we might put down as true "Copper conducts electricity" on the grounds that it is consistent with the hypothesis and affords it some particularising explanation. But for the very same reasons, we might put down as true the claim "Mercury conducts electricity". So should we put both of them down as true, on the basis of the indifference argument that, since there is not yet more reason to assert one rather than the other, and one needs to assert something in order to get anywhere, therefore one must assert both? No: there are reasons for thinking we ought not to assert both, on the present interpretation. For even though the claim about copper and the claim about mercury are perfectly consistent with one another, it is far from clear that they afford each other explanatory support of the sort I have been discussing, since neither is more general nor more particular than the other. They are, as it were, propositions "of the same level". There could only be collateral support between them. But it is doubtful that there is any such thing. It just does not seem to matter to the fact

${ }^{22}$ I do not of course mean that you will get a $\sigma v \mu \varphi \omega v i ́ \alpha$ if you sound C, G and D together. The tone interval between $\mathrm{C}$ and $\mathrm{D}$ will disrupt the purity of the fifth between $\mathrm{C}$ and $\mathrm{G}$. But if one goes from $\mathrm{C}$ to $\mathrm{G}$, and thence to $\mathrm{D}$, one always proceeds according to intervals of the right kind. This recalls the sense suggested at the end of Republic VI in which the hypothetical method involves somehow moving from one proposition to another in a sequence, a thing only dialectic does from a proper first principle.

23 I owe this observation to Ben Morison. 
that copper is a conductor, by itself, whether or not mercury is one as well. But if we take ourselves to be following Plato's hypothetical method in this set-up, it ought to matter. For Socrates says that you have finished developing your hypothesis only when you know that its results bear the right relation, not just to it, but to one another. All the o $\rho \mu \eta \theta \dot{\varepsilon} v \tau \alpha$ must

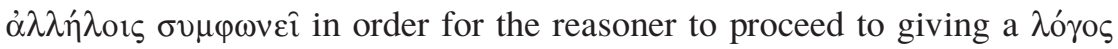
for the hypothesis itself.

This is a nettle I am prepared to grasp: it seems to me in the nature of the $\sigma 0 \mu \varphi \omega v \varepsilon i v$ relation that, at any stage, many candidates for satisfying it - p, q, r and so on - will emerge, only one of which can be selected at a time and put down as true, say p, on the grounds that the others, $q$ and $r$, do not cohere with $\mathrm{p}$ in the right way, even though they are consistent with it and cohere no less in the right way with the hypothesis. For anyone following Plato's method from the hypothesis that metals conduct electricity, he will have to decide which of the two particularising explanations to go for, assert that, and stay silent at least for the moment about the others, however strange that looks. Presumably, the idea would be to develop the hypothesis via one proposition, and then start again by putting down as true the other, and then comparing the degree to which the resulting sets of propositions form adequate theories of the kind the hypothetical method is after. It should not be a surprise by now that the same thing can happen in music. From the starting point $\mathrm{C}$, one will get a $\sigma v \mu \varphi \omega v i \alpha$ with the $\mathrm{F}$ a fourth above it, or alternatively the $\mathrm{G}$ a fifth above - both notes are suitably related to the original. But one cannot, as it were, go for both of these pitches together. For the $\mathrm{F}$ and the $\mathrm{G}$ do not blend into

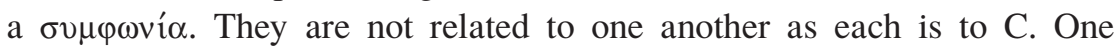
must choose one of them at a time if one intends to develop a musical complex of the right sort from $\mathrm{C}$, and there may at first be no more reason to choose one rather than the other. This strain of indeterminacy brings out the element of unexplained talent that will be required for a successful application of the method. Socrates has no story to tell about why you should put down as true one particular proposition from the many candidates that will emerge. But he does at least realise that the plurality of candidates is going to mean that one must pay special attention to the order in which one is proceeding - order forced upon the reasoner by the nature of the $\sigma u \mu \varphi \omega v \varepsilon i v v$ relation - perhaps so that the hypothesiser can effectively retrace his steps and start developing his hypothesis all over again with different propositions that $\sigma 0 \mu \varphi \omega v \varepsilon \hat{\imath}$ it without also bearing that relation to the first asserted batch of o $\rho \mu \eta \theta \dot{\varepsilon} v \tau \alpha$. 
I hope that this has gone some way to establishing my view that, for Plato, theory building is a kind of propositional orchestration. There is much of which I am uncertain, and much that this paper lacks the space to consider in detail, but I am convinced of the appropriateness of that general description. Of course the analogy goes lame in this respect: there are presumably better and worse ways of developing a hypothesis via the right coherence relations as described above, but it is presumably not the case that there are better and worse ways of following a starting pitch with the right concordant intervals. But nonetheless in developing the analogy we have found that the hypothetical method is much richer and more sophisticated than Socrates' brief sketch conveys, which is something we should prefer - especially given the reasons I have set out indicating that Plato has music in mind anyhow in this context. ${ }^{24}$

\section{Corpus Christi College \\ Oxford}

${ }^{24}$ I am very grateful to Nicholas Denyer and David Sedley, both of whom read this piece several times and saved me from many mistakes, as well as providing much helpful input and encouragement. But I am especially grateful to Andrew Barker, without whom it could not have been written at all. 